\title{
Reconstructing feedback representations in ventral visual pathway with a generative adversarial autoencoder
}

\author{
Haider Al-Tahan ${ }^{1,2}$ and Yalda Mohsenzadeh ${ }^{1,2,{ }^{*}}$
}

1 Department of Computer Science, The University of Western Ontario, London, Ontario, Canada

2 Brain and Mind Institute, The University of Western Ontario, London, Ontario, Canada

*ymohsenz@uwo.ca

\begin{abstract}
While vision evokes a dense network of feedforward and feedback neural processes in the brain, visual processes are primarily modeled with feedforward hierarchical neural networks, leaving the computational role of feedback processes poorly understood. Here, we developed a generative autoencoder neural network model and adversarially trained it on a categorically diverse data set of images. We hypothesized that the feedback processes in the ventral visual pathway can be represented by reconstruction of the visual information performed by the generative model. We compared representational similarity of the activity patterns in the proposed model with temporal (magnetoencephalography) and spatial (functional magnetic resonance imaging) visual brain responses. The proposed generative model identified two segregated neural dynamics in the visual brain. A temporal hierarchy of processes transforming low level visual information into high level semantics in the
\end{abstract}


feedforward sweep, and a temporally later dynamics of inverse processes reconstructing low level visual information from a high level latent representation in the feedback sweep. Our results append to previous studies on neural feedback processes by presenting a new insight into the algorithmic function and the information carried by the feedback processes in the ventral visual pathway.

\section{Author summary}

It has been shown that the ventral visual cortex consists of a dense network of regions with feedforward and feedback connections. The feedforward path processes visual inputs along a hierarchy of cortical areas that starts in early visual cortex (an area tuned to low level features e.g. edges/corners) and ends in inferior temporal cortex (an area that responds to higher level categorical contents e.g. faces/objects). Alternatively, the feedback connections modulate neuronal responses in this hierarchy by broadcasting information from higher to lower areas. In recent years, deep neural network models which are trained on object recognition tasks achieved human-level performance and showed similar activation patterns to the visual brain. In this work, we developed a generative neural network model that consists of encoding and decoding sub-networks. By comparing this computational model with the human brain temporal (magnetoencephalography) and spatial (functional magnetic resonance imaging) response patterns, we found that the encoder processes resemble the brain feedforward processing dynamics and the decoder shares similarity with the brain feedback processing dynamics. These results provide an algorithmic insight into the spatiotemporal dynamics of feedforward and feedback processes in biological vision. 


\section{Introduction}

In just a couple of hundred milliseconds, our brain interprets the visual scene around us [1 5], identifies faces [6, 7], recognizes objects [8 13], and localizes 3 them 14 18. Decades of cognitive neuroscience research has demonstrated that 4 the brain accomplishes these complicated tasks through a cascade of hierarchical 5 processes in the ventral visual stream starting in the early visual cortex (EVC) 6 and culminating in the inferior temporal (IT) cortex.

While the feedforward recruitment of this hierarchy explains the core neural 8 response patterns underlying visual recognition $[19$ 21], it is unable to account 9 for behavioural and neural dynamics observed in years of psychophysical, $\quad 10$ neurophysiological, and magneto/electrophysiological experiments $[12,22,25]$. 11 Indeed, variable timing of neural responses to visual stimuli beyond $200 \mathrm{~ms}$ has $\quad 12$ been frequently associated with accumulation of sensory evidence through ${ }_{13}$ recurrent processes in the visual brain. However, the precise computational role ${ }_{14}$ of neural recurrent/feedback processes remains poorly understood at the system ${ }^{15}$ level. In particular, the algorithmic function of feedback processes and the type 16 of information sent back along the visual hierarchy is still unknown. 17

To address this question, we develop a generative model which is 18 adversarially trained on a diverse set of image categories. The model consists of 19 two sub-networks: (i) An encoder sub-network receives a given visual stimulus, 20 processes it in a hierarchy of neural layers to eventually produce a latent $\quad 21$ representation (code) of the visual input and (ii) a decoder sub-network which $\quad 22$ receives the latent representation and aims to reproduce the visual input from ${ }_{23}$ the information encoded in the latent representation. 24

This generative model enables us to not only investigate the encoding process $\quad 25$ of visual representations along the hierarchy of the encoder sub-network layers $\quad 26$ but also provides us an insight into the reverse process i.e. reconstructing the ${ }_{27}$ representations along the decoder sub-network layers. We hypothesize that the ${ }_{28}$ 
visual information in the encoder sub-network in our computational model $\quad 29$ mimics the feedforward pathway in the ventral visual stream and the decoder 30 sub-network which performs the reverse function may reveal the representations 31 along the feedback pathway. To test this hypothesis, after training the proposed 32 model, we compare the representations along its layers with 33 magnetoencephalography (MEG) and functional magnetic resonance imaging $\quad 34$ (fMRI) data acquired from fifteen human participants in a visual recognition $\quad 35$ experiment 13].

Our model identified two separate dynamics of representational similarities $\quad 37$ with MEG temporal data. The first one is consistent with the temporal 38 hierarchy of processes transforming low level visual information into high level 39 semantics in the feedforward sweep, and the second one reveals a temporally $\quad 40$ subsequent dynamics of inverse processes reconstructing low level visual $\quad 41$ information from a high level latent representation in the feedback sweep. $\quad 42$ Further, comparison of encoder and decoder representations with two fMRI $\quad 43$ regions of interests, namely EVC and IT, revealed a growing categorical ${ }_{44}^{44}$ representation along the encoder layer (feedforward sweep) similar to IT and a ${ }_{45}$ progression in detail visual representations along the decoder layers (feedback $\quad 46$ sweep) akin to EVC.

\section{Results}

\section{Construction of a generative model performing image} reconstruction

Previous work revealed that deep convolutional neural networks (DNNs) trained 51 on image classification develop hierarchical representations similar to the $\quad 52$ cascade of processes along ventral visual pathway [3, 26 33]. However, 53 neuroscience evidence suggests top-down modulations of neural responses which $\quad 54$ occur after some delay through abundant number of feedback connections in $\quad 55$ 
visual cortex are critical to resolving visual recognition in the brain $12,23,24 . \quad 56$ Therefore, these feedforward deep neural network models do not fully represent $\quad 57$ the complex visual processes in the ventral visual pathway. Here, we investigate 58 whether a deep generative model trained to compress and reconstruct images $\quad 59$ could reveal similar representations as feedforward and feedback processes in the 60 ventral visual pathway. With this aim, we developed a deep generative $\quad 61$ autoencoder neural network model using adverserial autoencoder (AAE) 62 framework [34]. AAE is a generative adverserial network (GAN) [35] where the ${ }^{63}$ generator has an autoencoder architecture. Figure 1 depicts our proposed model $\quad 64$ architecture. The autoencoder generator consists of two main components: 1) 65 an encoder which receives the visual stimuli and performs a cascade of simple $\quad 66$ operations such as convolution, pooling, and normalization to map the visual $\quad 67$ input to a latent feature vector $(\mathrm{LV}) ; 2$ ) a decoder which receives the latent $\quad 68$ vector and performs a cascade of simple deconvolution, upsampling and $\quad 69$ normalization operations to reconstruct the input visual stimuli from $\quad 70$ information encoded in the latent space. The model is trained with two 71 objectives - a reconstruction loss criterion, and an adversarial criterion. The 72 dual objectives training turns the autoencoder into a generative model whose $\quad{ }_{73}$ latent space learns data distribution properties that enables generative process $\quad 74$ and avoids overfitting to the reconstruction objective. We hypothesize that the 75 encoder sub-network models the feedforward pathway of processes in ventral 76 visual stream, while the decoder sub-network models the reconstruction of visual 77 $\begin{array}{ll}\text { features in the feedback pathway. } & 78\end{array}$ \ To train our model, we assembled a super category data set (see Materials 79 and methods section for details). The super category data set includes 1,980,000 80 images from four equally distributed categories of (1) Faces, (2) Animates, (3) 81 Objects, and (4) Scenes. The rational behind assembling and using this data set 82 is two-fold: (1) ecologically, the human brain learns to develop high-level 83 category representations across multiple recognition tasks (e.g. faces, animals, ${ }_{84}$ 
objects, scenes, etc.); Indeed, years of neuroscience research have identified a $\quad{ }_{85}$ cascade of brain regions along ventral visual stream starting in early visual $\quad 86$ cortex (EVC) which processes low level visual features and eventually resolves $\quad{ }_{87}$ visual categories at the end of the hierarchy in inferior temporal cortex (IT). (2) 88 In this study, we compare model representations with brain imaging data (fMRI 89 and MEG) from a visual recognition experiment [13]. We maintained consistency 90 with the four categories from the stimulus set utilized in the brain imaging $\quad 91$ experiment which includes 156 images. Please note that this stimulus set was 92 not used for training the model. After training the model on super category $\quad 93$ data set for over 800 epochs when the adverserial and reconstruction losses $\quad 94$ reached their local minima on the training set (Figure 2A), we determined how 95 well our model performed on the 156 image set (as a testing dataset). Figure 2B 96 illustrates this image set and the corresponding reconstructed images. The 97 model performed the reconstruction on the training dataset with $0.109 \pm 0.006 \quad 98$ mean absolute error (MAE) and on the 156 image set with $0.119 \pm 0.003 \mathrm{MAE} \quad 99$ between the input image and the corresponding reconstructed image. We 100 computed the upper-bound performance of the model by sampling random pairs 101 of images from the training and testing sets performing MAE between the 102 different image pairs. On the training set, we observed a MAE of $0.53 \pm 0.014 \quad 103$ between the random image pairs and $0.62 \pm 0.017$ on the random pairs from the ${ }_{104}$ testing set. These results show that the model not only have converged to an $\quad{ }_{105}$ optimum but also generalizes well to the testing set.

\section{Representational similarity of the generative model to early and ${ }_{107}$ late brain regions in the ventral visual stream

We first determined the encoder/decoder representational similarities with early ${ }_{109}$ and late brain regions along the ventral visual cortex. For this, we chose early $\quad 110$ visual cortex (EVC) and inferior temporal cortex (IT) defined anatomically $\quad 111$ based on 36 and employed the representational similarity analysis (RSA) 112 


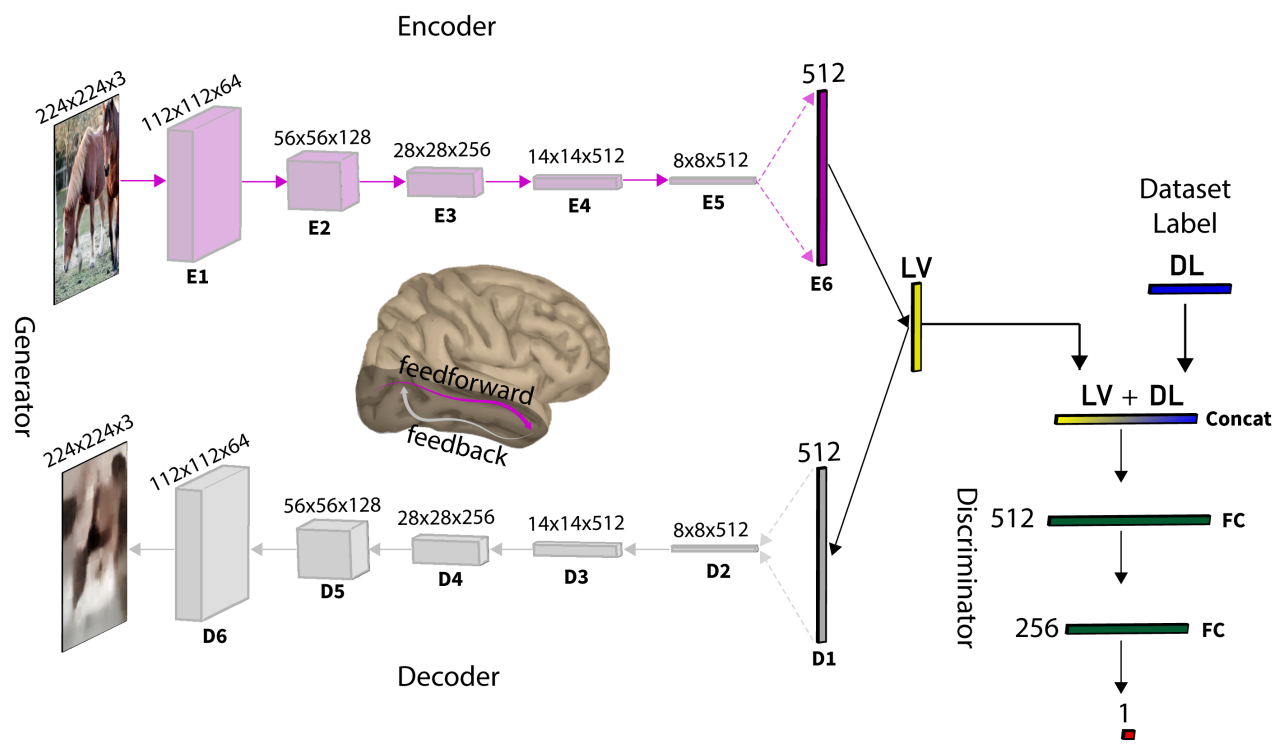

Fig 1. Computational model architecture. The model is a generative adverserial network. The generator is an autoencoder consisting of five convolutional blocks (E1-E5) and one fully connected layer (E6) in the encoder and one fully connected layer (D1) followed by five deconvolutional blocks in the decoder (D2-D6). Each convolutional block encompasses batch normalization, convolution, nonlinear activation function, and pooling operations. Alternatively, each deconvolutional block encompasses batch normalization, deconvolution, nonlinear activation function, and upsampling operations. The discriminator consists of two fully connected layers. The training Data set consists of 1,980,000 images organized into four super-ordinate categories: (i) Faces, (ii) Animates, (iii) Objects, (iv) Scenes. LV denotes the latent vector generated by the encoder and DL is a one-hot data set label (one of the four mentioned training data sets). Both vectors are concatenated and fed to the discriminator, while only the latent vector is fed to the decoder.

method 37,38 as the integrative framework for model-brain comparisons.

For each region of interest (ROI), we extracted the fMRI response patterns to each image, vectorized it and computed condition-specific pairwise distances (1114 Pearson's R) to create a 156 x 156 representational dissimilarity matrix (RDM) per participant. We also fed the images to the generator and extracted 117 layer-specific activations for each image condition. Then by computing the ${ }_{118}$ pairwise distances (1-Pearson's R) of image evoked layer activation patterns, we ${ }_{119}$ created layer-specific RDMs (see Figure 3 and Materials and methods section for ${ }_{120}$ details). We then compared subject-specific ROI RDMs with the model layer ${ }_{121}$ 
A

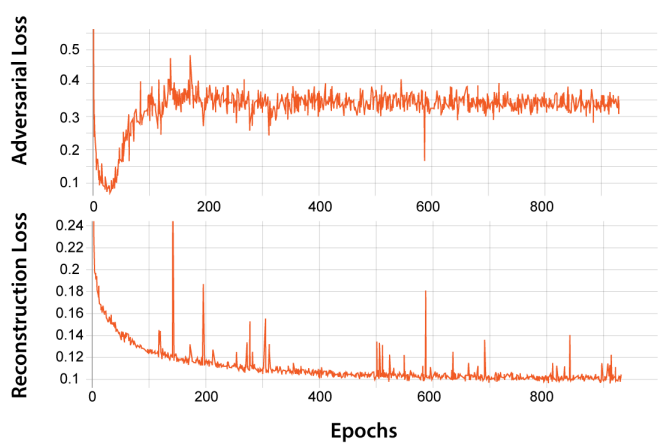

B
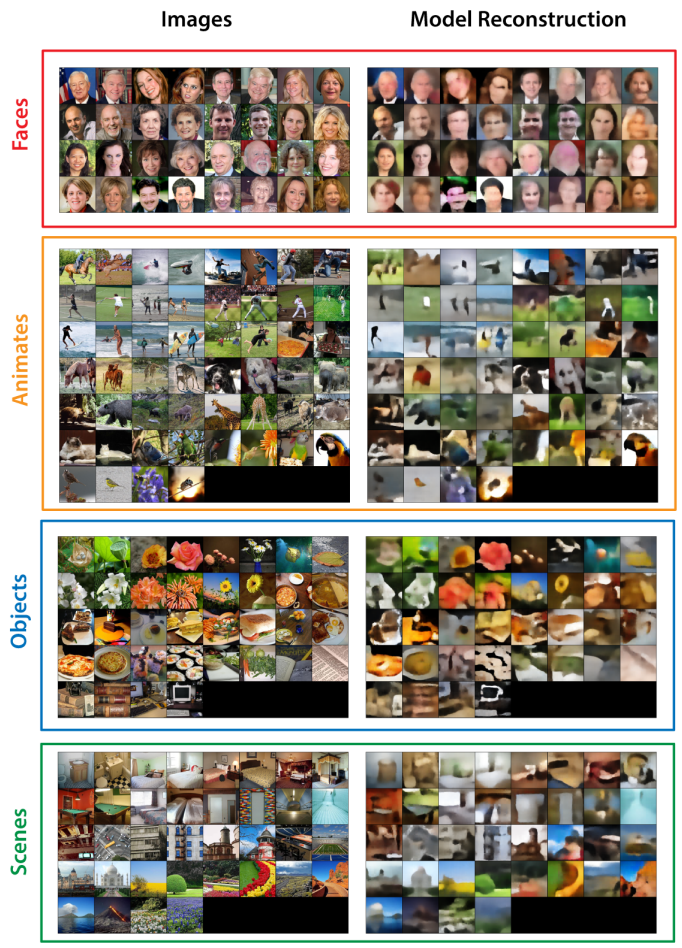

Fig 2. Computational model performance. (A) Adversarial and Reconstruction loss over epochs. (B) 156 image set and their corresponding reconstructed image by the model.

RDMs by computing Spearman's correlations (Figure 3ADE). Figure 4A and B ${ }_{122}$ show subject averaged RDMS and their corresponding 2-dimensional multidimensional scaling (MDS) visualizations of EVC and IT, respectively. As ${ }_{124}$ expected EVC shows a random pattern across categories, whereas IT 125 demonstrates clear categorical distinctions. Figure $4 \mathrm{C}-\mathrm{D}$ compares the encoder ${ }_{126}$ and decoder layers correlations with EVC and IT RDMs. 127

The EVC representational correlations across layers of the encoder (and $\quad{ }_{128}$ 
inverse order of the decoders) demonstrate a decreasing trend, while IT $\quad 129$ representational correlations across model layers progressively increase. Notably, 130 early processing layers of the model (E1/D6) and late processing layers (E6/D1) 131 show similar correlations both for EVC and IT. However, middle layers 132 correlations are significantly different when comparing encoder and decoder in $\quad 133$ both ROIs $(\mathrm{N}=15$; two-sided ttests; false discovery rate corrected at $P<0.05)$. ${ }_{134}$ Further, the correlations of EVC is stronger with the decoder than encoder $\quad 135$ whereas the correlations of IT is higher for the encoder than the decoder. This ${ }_{136}$ indicates the reconstructing processes are more similar to detail representations $\quad{ }_{137}$ in EVC than IT. 138

\section{The generative model unfolds the temporal dynamics of brain $\quad{ }_{139}$} feedforward and feedback representations

The visual information traverses a hierarchy of regions in the visual cortex and ${ }_{141}$ evolves over time rapidly. While the structure of our proposed model does not ${ }_{142}$ directly delineate the human brain temporally, it has a clear sequential structure ${ }_{143}$ which temporally unfolds the feedforward and feedback sequences in the visual ${ }_{144}$ cortex. That is, information flows layer to layer and evolves from image low $\quad 145$ level features to higher level latent concepts (feedforward sweep) and then from ${ }_{146}$ this high level latent code sequentially the image level information is $\quad 147$ reconstructed (feedback sweep). To test the hypothesis that the proposed model ${ }_{148}$ indeed temporally mirrors ventral visual stream dynamics, we compared the $\quad{ }_{149}$ representations of time-resolved MEG data acquired in a visual recognition $\quad 150$ experiment with the layer representations in the encoder and decoder 151 sub-networks. From each participant's data, we first extracted the MEG sensor 152 measurements for each image condition from -200ms to $1000 \mathrm{~ms}$ (with $1 \mathrm{~ms} \quad 153$ resolution) relative to image onset. Then we computed dissimilarities (SVM $\quad 154$ classifiers decoding performances, see Materials and methods section for details) ${ }_{155}$ between evoked MEG pattern vectors of each pair of images and created time $\quad{ }_{156}$ 


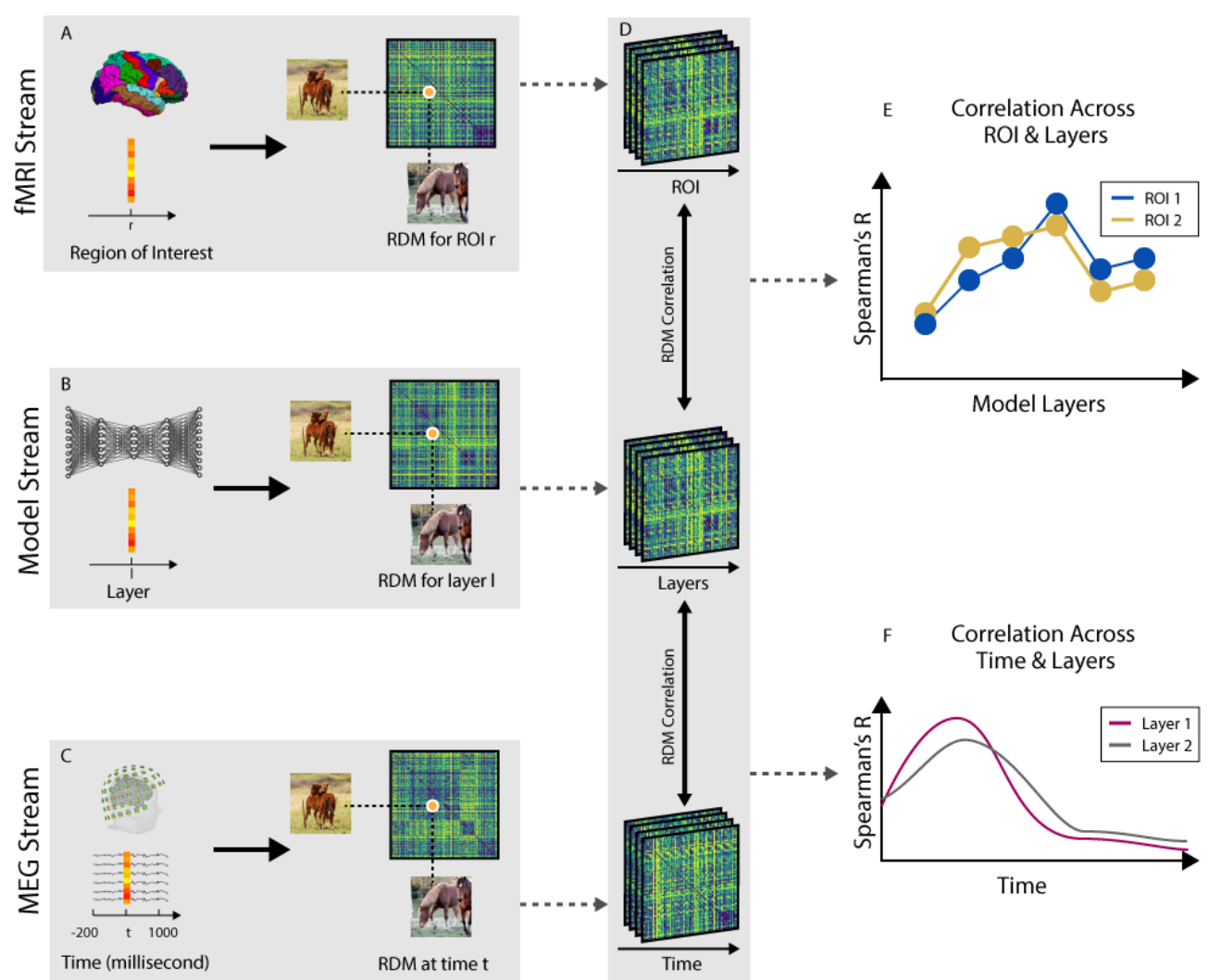

Fig 3. Representational similarity analysis to compare fMRI, MEG and model representations. (A) fMRI response patterns were extracted from each ROI and pairwise condition-specific dissimilarities (1-Pearson's R) were computed to create one fMRI RDM per ROI and participant (see Materials and methods section for detail). (B) RDMs for the generative model were computed at each convolutional/deconvolutional block after feeding 156 images to the computational model. (C) MEG data consists of time-series data with 306 channels and 1200 time points (milliseconds) per trial. For each condition, we extracted a vector of size 306 at each time point as the activity pattern to compute the RDMs using SVM classifiers decoding accuracies (see Materials and methods section for detail). (D) Using RDMs from MEG and fMRI ROIs, we compared (Spearman's R) them with the RDMs from the computational model to investigate the spatio-temporal correspondences between the human brain and the computational model. (E) Correlations between time-resolved MEG RDMs and computational model RDMs result in a subject-specific signal for each layer across time, which we then average them over subjects.

resolved representational dissimilarity matrices (RDMs) for each individual. We 157 also fed the images to the generator and extracted layer-specific activations for ${ }_{158}$ each image condition. Then by computing the pairwise distances (1-Pearson's R) 159 of image evoked layer activation patterns, we created layer-specific RDMs (see $\quad 160$ 

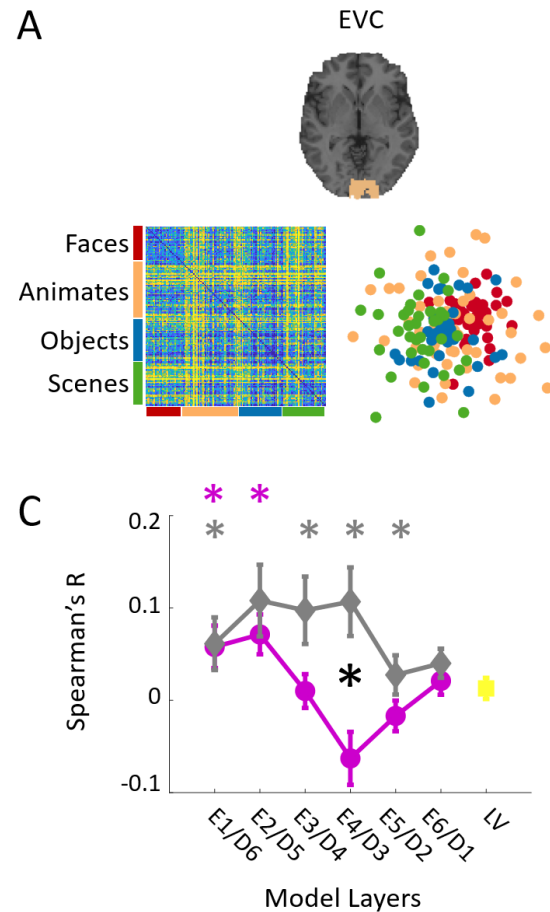

B
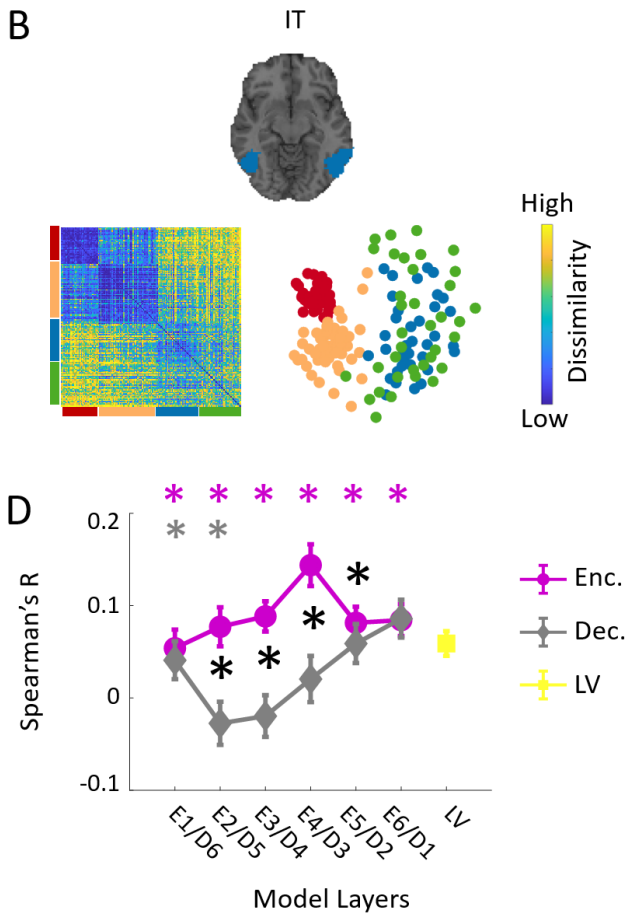

Fig 4. Spatial representational comparisons (A) Neural representations in early visual cortex (EVC). The subject-averaged EVC RDM matrix, and its 2D multidimensional scaling visualization. (B) Neural representations in inferior temporal area (IT). The subject-averaged IT RDM matrix, and its 2D multidimensional scaling visualization. (C) Encoder, decoder and LV layer RDMs are correlated (Spearman's R) with subject-specific EVC RDMs. The averaged correlations over subjects with standard error of the mean are depicted. (D) Encoder, decoder and LV layer RDMs are correlated (Spearman's R) with subject-specific IT RDMs. The averaged correlations over subjects with standard error of the mean are depicted. The color coded $\left(^{*}\right)$ above each panel in C-D indicates that the correlation of the corresponding layer is significantly above zero. The black $(*)$ indicates the correlations of the corresponding encoder and decoder layers are significantly different $(\mathrm{N}=15$; two-sided ttests; false discovery rate corrected at $P<0.05$ ).

Figure 3 and Materials and methods section for details). Then we correlated ${ }_{161}$ layer-specific model RDMs with time-resolved subject specific MEG RDMs $\quad{ }_{162}$ (Spearman's R) resulting in correlation time series for each layer of the model. $\quad{ }_{163}$ Figure 5A-B show the subject averaged correlation time series for layers of $\quad 164$ encoder and decoder sub-networks, respectively. Our results showed that all $\quad 165$ layers of the proposed model were representationally similar to human brain $\quad{ }_{166}$ 
activity patterns, indicating that the model captures evolution of brain visual $\quad{ }_{167}$ representations over time $\left(\mathrm{N}=15\right.$;permutation tests; cluster definition threshold ${ }_{168}$ $P<0.05$; cluster significance threshold $P<0.01)$. Next, we investigated $\quad 169$ whether the hierarchy of the layered architecture unfolds the temporal dynamics 170 of encoding (feedforward) and decoding (feedback) visual processes in the brain. 171 Specifically, we examined the relationship between hierarchy of model layers and 172 the peak latency of the correlation time series (Figure $5 \mathrm{C}$ ). Consistent with $\quad 173$ previous works [29], the first peak latency of correlation time courses relating $\quad 174$ MEG and the encoder representations increased with the hierarchy of the $\quad 175$ encoder layers (Spearman's $R=0.78, P<<0.0001$ ). The inspection of peak 176 latencies in the decoder time series depicted in Figure $5 \mathrm{~B}$ revealed a 177 progressively increasing pattern from D1 to D6 (Spearman's $R=0.84, \quad 178$ $P<<0.0001)$. That is, the peak latency in D1 occurs at $151 \mathrm{~ms}$ and over the ${ }_{179}$ decoder layers progressively the peak latency increases, the peak latency of D6 180 occurs at 204ms. Given that the decoder later layers are reconstructing the $\quad 181$ visual stimuli and thus closer to image level feature space, these temporal $\quad{ }_{182}$ dynamics may explain the temporal dynamics of feedback information sent $\quad 183$ down the ventral stream. We further identified a salient second peak in layers $\quad{ }^{184}$ E1 to E5 which their peak latencies negatively correlated with layers order in $\quad{ }_{185}$ the Encoder (Spearman's $R=-0.57, P<<0.0001$ ) and they are significantly 186 later than the corresponding layers in the decoder (all peak latency analyses are ${ }_{187}$ based on permutation based bootstrapping; $\mathrm{N}=15$; two-sided hypothesis tests; $\quad{ }_{188}$ $P<<0.0001$; Bonnferoni corrected). Again confirming a later dynamics of $\quad 189$ representational similarities between these layers and the brain, possibly $\quad 190$ indicating a second sweep of visual information processing. 191

In the next step, we investigated the representational relationships among the 192 encoder and the decoder layers of the model. To this end, we first computed the ${ }_{193}$ pairwise correlations between all encoder and decoder layer RDMs. The matrix 194 depicted in Figure 5 E summarizes these pairwise correlations and reveals which 195 
representations across model layers are similar or dissimilar. Figure $5 \mathrm{~F} \quad 196$ visualizes these relationships with multi dimensional scaling (MDS) method. $\quad{ }_{197}$ Visual inspection of this matrix manifests firstly the dissimilarity of latent layer ${ }_{198}$ representation from encoder and decoder layers, secondly similarity of late layer ${ }_{199}$ of encoder (E6) and early layer of the decoder (D1); and also similarity of early 200 layer of decoder (E1) and late layer of the decoder (D6), thirdly the dissimilarity 201 of middle layers of encoder and decoder indicating the difference in the 202 representations of encoding (feedforward) and decoding (feedback) processes. $\quad 203$

To obtain a more clear picture of brain-model temporal dynamics 204 relationships across encoder and decoder sub-networks, we compared the 205 correlation time courses corresponding to the encoder and decoder layers with $\quad 206$ the same level of processing in Figure 6A. The correlation time course of latent 207 layer is depicted separately. Figure 6B depicts the corresponding model layers 208 RDMs and their 2-dimensional MDS visualizations. As demonstrated in Figure 209 5. firstly the low level feature processing layer of encoder (E1) and decoder (D6) ${ }_{210}$ follow a notably similar dynamics. Further, the high level feature processing $\quad 211$ layers of encoder (E6 and E5) also depict a similar temporal dynamics with high 212 level feature processing layers of the decoder (D1 and D2, respectively). ${ }^{213}$ However, the dynamics are explicitly different when middle level layers are $\quad 214$ compared (i.e. E2 vs.D5, E3 vs. D4, E4 vs. D3). This is also evident from 215 comparison of the MDS visualizations of RDMs in each row. The peak latencies 216 of the correlation time courses are marked with color-coded arrows. Consistent ${ }_{217}$ with Figure $5 \mathrm{C}$, the peak latencies of low and high level processing layers $\quad{ }_{218}$ coincide around the same time, whereas the peaks of mid-level feature ${ }_{219}$ processing layers of the decoder occurs between the first and second peaks of the 220 corresponding encoder time courses. 221

Together, comparison of MEG temporal representations with the encoder and 222 decoder sub-networks of our proposed model segregated the brain ${ }_{223}$ representational dynamics that transforms the low level visual features to high $\quad 224$ 
level categorical semantics and the inverse functional processes that reconstructs 225 low level features from the high level code. These two identified dynamics of $\quad 226$ processing can be associated with feedforward and feedback sweeps along ventral ${ }_{227}$ visual stream.

\section{Discussion}

Using a generative model, we dissected the dynamics of processes in the ventral 230 visual pathway into two temporally distinct stages: the initial sweep depicts $\quad 231$ neural representational similarity with the hierarchy of representations along the 232 encoder sub-network which transforms visual features into a latent 233 representation (feedforward sweep); and the subsequent sweep shows neural 234 representational similarity with the decoder sub-network which layer by layer $\quad 235$ reconstructs visual details from the latent representational code (feedback 236 sweep).

As demonstrated in Figure [5A-C, the temporal representational similarities ${ }_{238}$ of the encoder sub-network replicates previous findings showing hierarchical $\quad 239$ representational similarities between the visual brain and the feedforward DNNs 240 that are trained on object recognition tasks $21,26,27,29,32,39$. Specifically, it 241 has been demonstrated that object recognition DNNs develop internal 242 representations that are hierarchically similar to brain representations in early 243 visual cortex [39], area V4 (a mid-level region), and IT cortex (a high level 244 categorical region) along ventral visual pathway in primates 21,26 and 245 humans 27,29, 32]. Unlike the object recognition DNNs, the encoder 246 sub-network in our model is trained in an unsupervised manner, i.e., the 247 information regarding image classes (e.g. a car, a basketball) were not provided 248 to this network during training. This is a critical finding, as it shows supervision 249 is not required for the development of this hierarchical architecture observed in 250 DNNs and the brain. Further, comparing the representational dynamics of the ${ }^{251}$ 


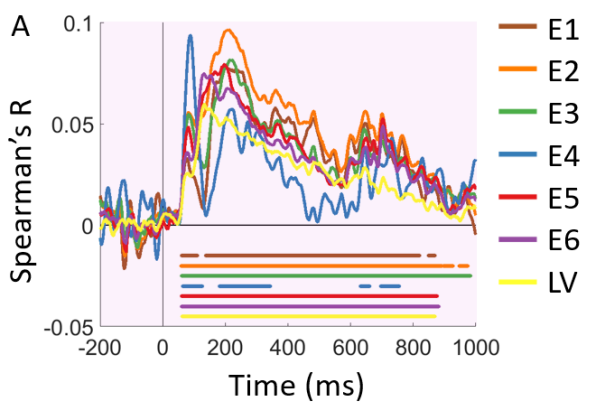

C

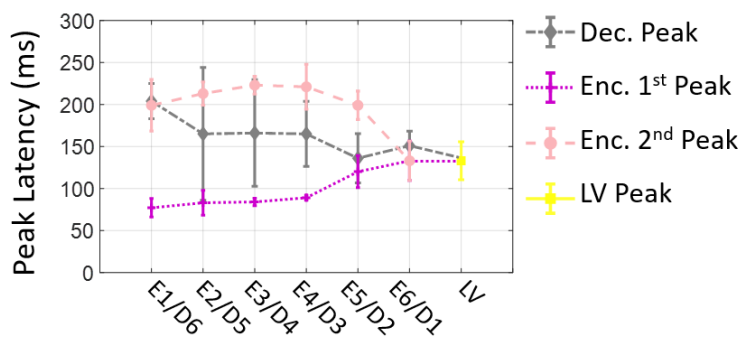

Model layers

D

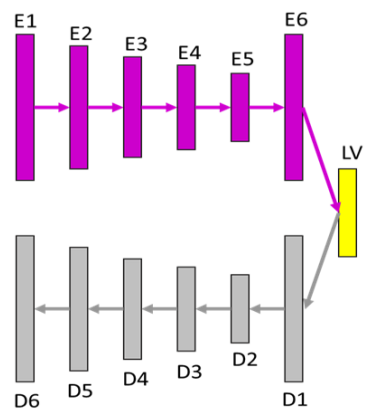

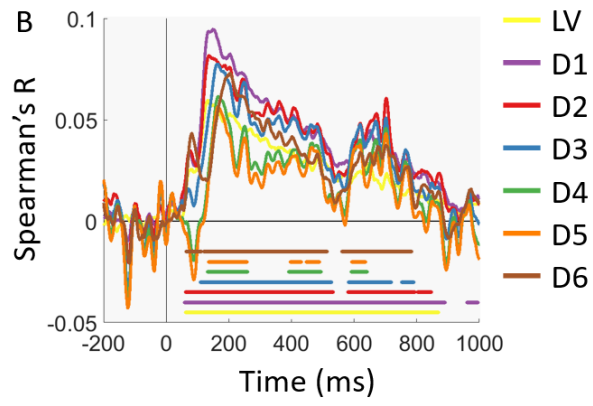

$E$

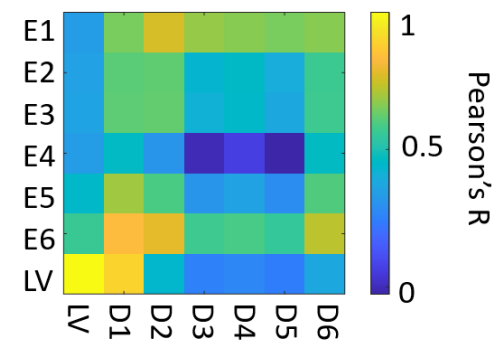

$\mathrm{F}$

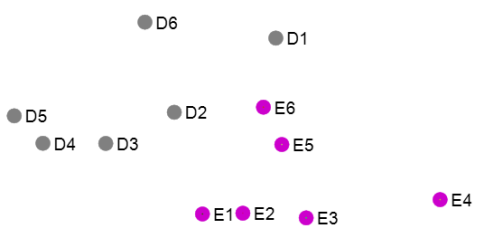

Fig 5. Temporal representational comparisons. (A) Encoder and MEG representational comparison. We correlated the encoder layer RDMs with subject-specific time-resolved MEG RDMs resulting in fifteen correlation time courses. We then averaged these time courses over participants. (B) Decoder and MEG representational comparison. Correlation of the decoder layer RDMs and time-resolved MEG RDMs. The color-coded lines below the curves show the time points when the correlations are significantly above zero $(\mathrm{N}=15$; permutation tests; cluster definition threshold $P<0.01$; cluster threshold $P<0.05)$. (C) Peak latency for encoder and decoder. The encoder have significantly earlier peak latency across all layers $(P=0.014)$. Error bars are expressed in standard error of the mean. (D) The architecture of the models with layers's label corresponding to (C). (E) The visualization of relationships between model layers representations. The matrix of RDM correlations between encoder and decoder layers is depicted. Each matrix entry compares two RDMs indexed by corresponding row and column in terms of Pearson's R. (F) The multidimensional scaling visualization of the RDMs relationships. 


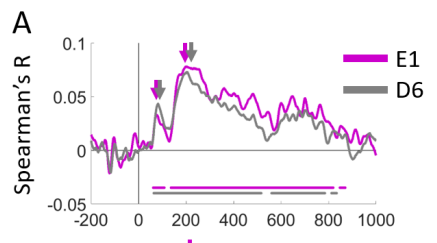

B
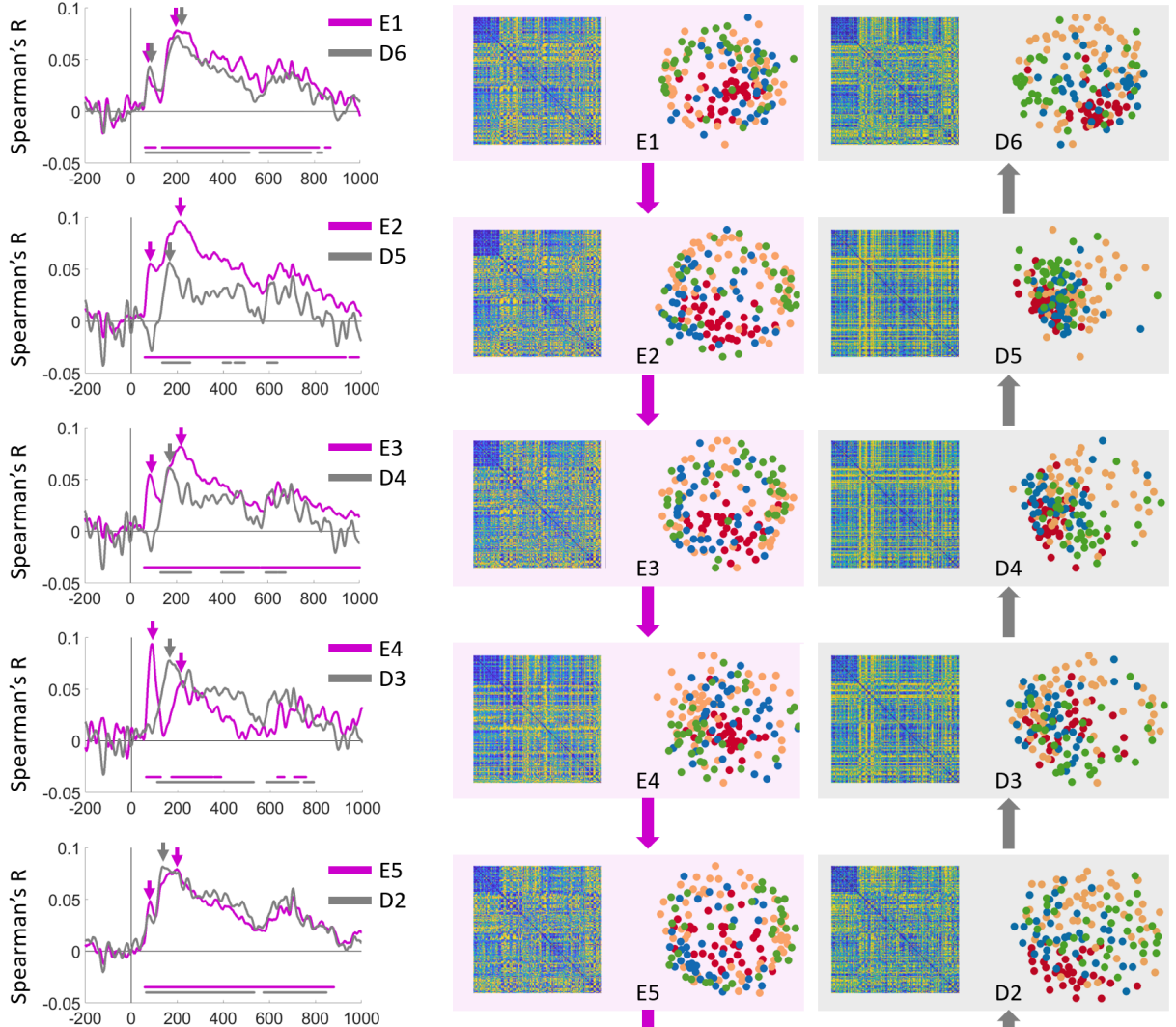

E2
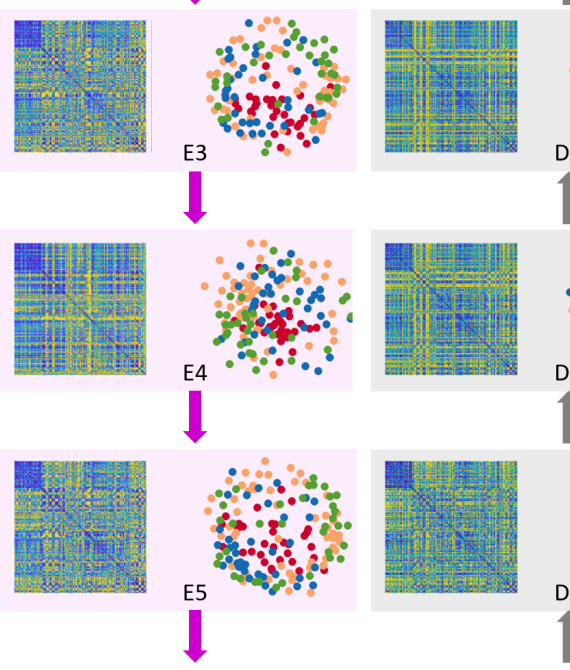

D5
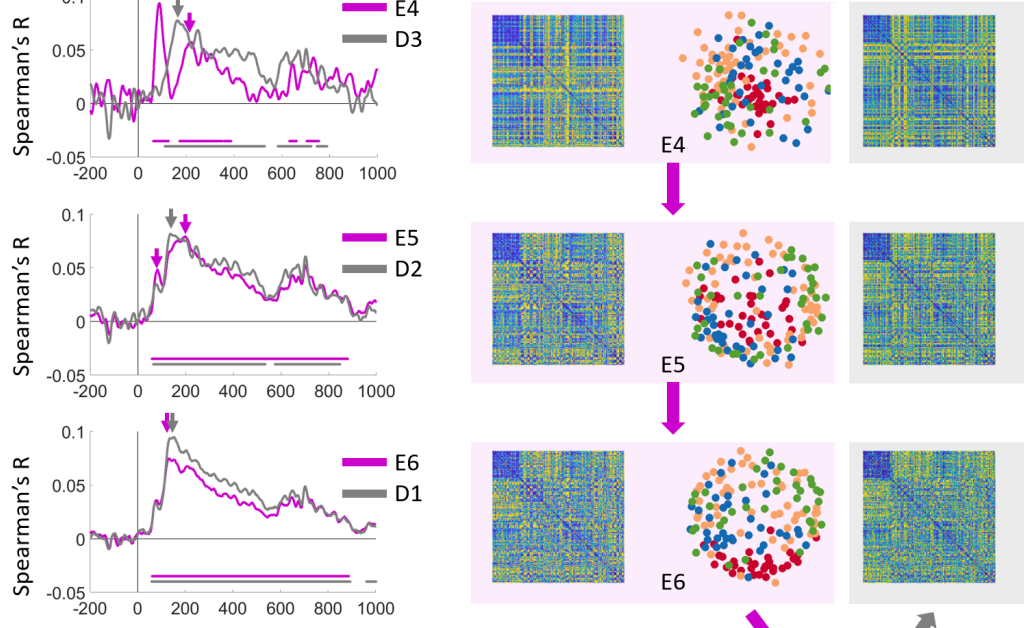

D4
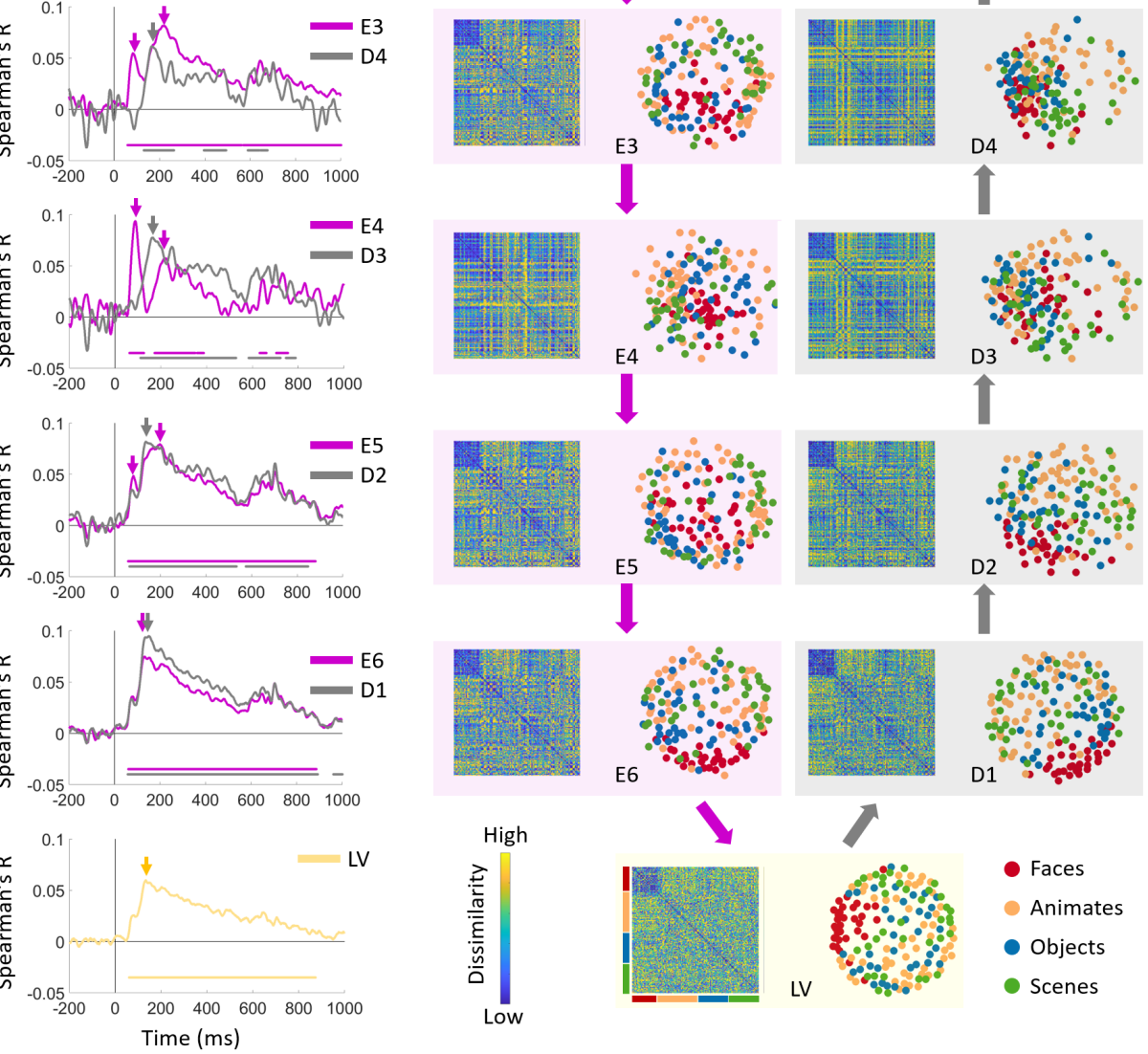

Fig 6. Comparisons of encoder and decoder representational dynamics. (A) Comparison of correlation time series of the encoder and decoder layers with the same level of processing. The color-coded lines below the curves show the timepoints when the correlations are significantly above zero (N=15; permutation tests; cluster definition threshold $P<0.01$; cluster threshold $P<0.05$ ). (B) The model RDMs and their corresponding MDS visualizations.

decoder sub-network and the visual brain revealed a temporally subsequent $\quad 252$ hierarchy of processing (Figure $5 \mathrm{~B}-\mathrm{C}$ ) which progressively builds visual detail ${ }_{253}$ 
from the latent representation. This indicates that beyond feedforward sweep, $\quad 254$ brain visual processes demonstrate similarities with the reconstruction function 255 implemented in the decoder sub-network. This finding contributes to unraveling 256 the algorithmic functional role of feedback processes in the visual cortex. $\quad 257$ Finally, representational comparison of two brain regions at the beginning and 258 end of ventral visual pathway, EVC and IT, with the encoder and decoder $\quad 259$ subnetworks (Figure 6) revealed the encoding processes in the feedforward sweep 260 develops a categorical representations similar to IT and the reconstructing $\quad 261$ processes in feedback sweep evolves into detail representations similar to EVC. $\quad 262$

\section{Materials and methods}

Our study consists of two components: (i) The computational model and (ii) the ${ }^{264}$ MEG/fMRI data from human participants, both of which are analyzed and $\quad 265$ compared in the result section. We focus on the ventral visual pathway, hence, ${ }^{266}$ we acquire both human brain and computational model neuronal activations on 267 visual centric tasks. In this section, we will describe the computational model, ${ }_{268}$ SP (Super Category) image dataset, and MEG/fMRI data acquisition and $\quad 269$ analysis.

\section{Neuroimaging experiments}

The fMRI and MEG data used in this study has been published in 13 analysis.

\section{Participants}

Brain data were acquired from fifteen right-handed healthy participants with $\quad 277$ normal or corrected to normal vision in two separate experiments (MEG and 
fMRI). Participants (9 females, $27.87 \pm 5.17$ years old) signed an informed $\quad 279$ consent form and were compensated for their participation. Both experiments 280 were conducted in accordance with the Declaration of Helsinki and approved by ${ }_{281}$ the Institutional Review Board of Massachusetts Institute of Technology. $\quad 282$

\section{Stimulus set and experimental design}

The stimulus set consists of 156 natural images of four distinct visual categories: ${ }^{284}$ (i) Faces, (ii) Animates (animals and people), (iii) Objects, (iv) Scenes 13 . The ${ }_{285}$ participants viewed images presented for $0.5 \mathrm{sec}$ (with $2.5 \mathrm{sec}$ inter stimulus $\quad 286$ interval (ISI) in fMRI sessions and 0.7 to 0.8 sec ISI in MEG session) at the $\quad{ }_{287}$ center of the screen at $6^{\circ}$ visual angle. Functional MRI data were acquired in $\quad 288$ two sessions (11-15 runs in total) and MEG data were acquired in one session of 289 25 runs. Images were presented once in each run and in random order. The 290 participants were performing a vigilance task of oddball detection.

The fMRI experiment was conducted at the Athinoula A. Martinos Imaging 293 Center at MIT, using a 3 T Siemens Trio scanner with 32-channel phased-array 294 head coil. Each imaging session started with acquiring structural images using a 295 standard T1-weighted sequence (176 sagittal slices, FOV $=256 \mathrm{~mm} 2, \mathrm{TR}={ }_{296}$ $2530 \mathrm{~ms}, \mathrm{TE}=2.34 \mathrm{~ms}$, flip angle $=9^{\circ}$ ) and then $5-8$ runs of 305 volumes of $\quad{ }_{297}$ functional data (11-15 runs across the two sessions). Gradient-echo EPI 298 sequence was used for functional data acquisition $(\mathrm{TR}=2000 \mathrm{~ms}, \mathrm{TE}=29 \mathrm{~ms}, \quad 299$ flip angle $=90^{\circ}$, FOV read $=200 \mathrm{~mm}$, FOV phase $=100 \%$, bandwidth $2368 \quad 300$ $\mathrm{Hz} / \mathrm{Px}$, gap $=20 \%$, resolution $=3.1 \mathrm{~mm}$ isotropic, slices $=33$, ascending $\quad 301$ interleaved acquisition). For preprocessing of fMRI data, we used SPM software. 302 The preprocessing of functional data included slice-time correction, realignment 303 and co-registration to the first session T1 structural scan, and normalization to 304 the standard MNI space. For multivariate analysis, we did not smooth the data. 305 
We used general linear modeling (GLM) to estimate fMRI responses to the $156 \quad 306$ stimuli. The events including stimuli conditions and nulls were modeled with $\quad 307$ event onsets and impulse response function. Further, the motion and run 308 regressors were included in the GLM. Then we convolved the defined regressors 309 with the hemodynamic response function and estimated the beta-values for each 310 stimulus condition. Then by contrasting each image condition with the explicitly 311 defined null condition, we obtained t-mpas per image condition for each 312 participant. For the current study, we investigated two anatomically defined [36] 313 regions of interest (ROIs) along the ventral visual stream, early visual cortex $\quad 314$ (EVC) and inferior temporal cortex (IT). 315

We used multivariate analysis and computed pairwise dissimilarities between 316 156 image specific fMRI responses using 1-Pearson correlation distances and 317 constructed a 156 x 156 representational dissimilarity matrix (RDM) per 318 participant per ROI (EVC and IT). In detail, we extracted t-value patterns 319 corresponding to each image condition from each region of interest, arranged 320 them into vectors. Then we calculated the pairwise distances of the 156 image ${ }_{321}$ specific vector patterns. With this process, we obtained a 156 x156 RDM per ${ }_{322}$ ROI for each participant. 323

\section{MEG data acquisition and analysis 324}

The MEG experiment was conducted at the Athinoula A. Martinos Imaging 325 Center at MIT, using a 306-channel Elekta neuromag TRIUX system with 326 sampling rate of $1 \mathrm{kHz}$. The acquired data were filtered by a 0.03 to $330 \mathrm{~Hz} \quad 327$ band-pass filter. We measured the participants' head position prior and during 328 the recording with 5 coils attached to their head. We then applied a maxfilter ${ }_{329}$ for temporal source space separation and head movements correction 40, 41. $\quad 330$ For preprocessing of MEG data, we used Brainstorm software [40]. We extracted 331 trials from $-200 \mathrm{~ms}$ to $1000 \mathrm{~ms}$ with respect to image onset. We then removed 332 the baseline mean and smoothed the data with a $30 \mathrm{HZ}$ low-pass filter. For each 333 
participant, we obtained 25 trials per image condition. We employed 334 multivariate pattern analysis to compute the dissimilarity relations between $\quad 335$ image conditions $10,13,29,42,43]$. At each time point t, we arranged MEG 336 sensor measurements of each image condition into pattern vectors of $306 \times \mathrm{N} \quad 337$ dimension, where $\mathrm{N}$ denotes the number of trials per condition. We then 338 randomly assigned the trials of each condition into 8 bins and subaveraged the $\quad 339$ trials within each bin to overcome computational complexity and reduce noise. 340 Support vector machine classifiers were trained on the subaveraged MEG 341 pattern vectors of each pair of images at each time point to discriminate the ${ }_{342}$ pairs. The performance of the classifier in discriminating each pair of images ${ }_{343}$ with leave-one-out cross validation procedure was used as the dissimilarity $\quad 344$ measure between the pairs to populate a $156 \times 156$ representational dissimilarity 345 matrix $(\mathrm{RDM})$ at each time point. The rows and columns of the RDM are $\quad 346$ indexed by the image conditions and each matrix element indicates the 347 dissimilarity of the corresponding image conditions based on MEG 348 measurements of the specific time point. 349

\section{Computational Model Architecture and Training 350}

Previous work revealed that deep convolutional neural networks trained on 351 object recognition develop similar representations akin to the hierarchical 352 processes along ventral visual stream $[3,26,32$. However, there are abundant 353 number of feedback connections in ventral visual stream and therefore, these $\quad 354$ feedforward neural network models may not fully represent the complex visual 355 processes in the ventral visual pathway. 356

Here, we aim to investigate whether a deep generative model trained to map 357 images to a latent code and then reconstruct the images from the features 358 encoded in the latent space can reveal similar representations as feedforward and 359 feedback processes in the ventral visual pathway. With this aim, we developed a 360 deep generative autoencoder neural network model using adverserial 361 
autoencoder (AE) framework [34, 35].

AEs consist of two sub-networks: (i) The encoder takes a given data and

outputs a lower representation of the data (latent code) and (ii) the decoder $\quad 364$ takes the latent code and aims to reproduce the input data. Alternatively, the 365 GAN framework is a min-max adversarial game between two distinct neural 366 networks: (i) The generator $(G)$, aims at generating synthetic data by learning 367 the distribution of the real data and (ii) the discriminator $(D)$, aims at $\quad 368$ distinguishing the generator's fake data from real data. The generator uses a 369 function $G(z)$ that maps samples $z$ from the prior $p(z)$ (normal distribution) to 370 the data space $p(x) . G(z)$ is trained to maximally confuse the discriminator into 371 believing that samples it generates come from the data distribution. The 372 solution to this game can be expressed as following [35]:

$$
\min _{G} \max _{D}\left[\mathbb{E}_{x \sim p_{\text {data }}}[\log D(x)]+\mathbb{E}_{z \sim p_{z}}[\log (1-D(G(z)))]\right]
$$

We chose an AE architecture because we hypothesize that the encoder $\quad 374$ embodies similar neuronal characteristics as the image classification DNNs and 375 thereby could resemble the human brain feedforward representations. 376 Alternatively, we hypothesize that the decoder part of the AE architecture which 377 generates the image from the latent space code would encompass the neuronal 378 representations similar to feedback processes in the human visual brain. $\quad 379$

Our generative autoencoder model architecture consists of a total of 13 layers: 380 (i) the encoder consists of 6 layers; 5 convolutional layers and one 381 fully-connected layer, (ii) the decoder consists of 6 layers; 5 deconvolutional 382 layers and one fully-connected layer, and (iii) lastly one fully-connected layer $\quad 383$ representing the latent code layer The latent code vector z captures high level 384 representation of the data distribution. The discriminator architecture consists 385 of a total of three fully connected layers (Figure $1 \mathrm{~B}$ ). 386 


\section{Super Category (SC) Dataset}

In computer vision literature, deep neural network models are usually trained to 388 optimize category specific recognition performance on large scale datasets. $\quad 389$ However, the human brain learns to develop high-level representations for $\quad 390$ categories across multiple recognition tasks (eg faces, objects, scenes, etc). 391 Indeed, years of cognitive neuroscience has demonstrated brain regions which 392 are functionally respond preferentially to one of these categories compared to 393 others (eg. Fusiform gyrus, IT area, Parahipocampal cortex, ...). Therefore, to 394 train our proposed model we put together a super category (SC) data set 395 consisting of 1,980,00 images from four equally distributed distinct categories: 396 (i) Animals, (ii) Objects, (iii) Scenes, and (iv) Faces. Images from the Faces 397 category were acquired from the VGGFaces2 dataset [44], Objects and Animals 398 categories were from the ImageNet dataset [45], and Scenes categories were from 399 the Places356 dataset [46]. To make all classes equal, we have randomly sampled 400 495,000 images per class. During computational model training and testing, 401 each image was preprocessed through a pipeline: (i) Images were resized to $\quad 402$ $224 \times 224$, and (ii) normalized from 0 to 225 to -1 to 1 range values. 403

Lastly, the neuronal representations for the generative AE model were 404 computed at each convolutional/deconvolutional block after feeding 156 images 405 used in the neuroimaging experiments to the encoder of the computational $\quad 406$ model. Please note this image set was not used in training the model. We ${ }_{407}$ employed the vectorized model activity patterns of each 408 convolutional/deconvolutional block to compute dissimilarity distances $\quad 409$ (1-Pearson's R) for each pair of imagesand create an RDM per model layer $\quad{ }_{410}$ (Figure 3B). 411 


\section{Representational similarity analysis to relate the brain and $\quad 412$ model representations

We used representational similarity analysis (RSA) [37,38 to map MEG 414 measurements, fMRI responses, and model activation patterns into a common $\quad{ }_{415}$ space where they are directly comparable. $\quad 416$

RSA transforms the stimulus-specific response patterns into a 417 representational space by creating matrices summarizing pairwise distance $\quad{ }_{418}^{418}$ relationships of the response patterns (i.e. defined as the correlational distance, 419 or a classifier performance in discriminating two conditions). The matrix $\quad 420$ capturing these pairwise dissimilarity measures is called representational $\quad{ }_{421}$ dissimilarity matrix (RDM). $\quad 422$

To relate the spatio-temporal dynamics of neural representations in the $\quad{ }^{423}$ human brain with our proposed model, we computed the similarity (in terms of ${ }_{424}$ Spearman's R) of fMRI ROI RDMs and time-resolved MEG RDMs with our $\quad{ }^{425}$ computational model RDMs (Figure 3D). 426

Correlations between subject-specific time-resolved MEG RDMs and $\quad 427$ computational model layer RDMs result in a signal for each layer per participant $\quad 428$ across time (Figure 35). While, correlations between subject-specific fMRI ROI ${ }_{429}$ RDMs and computational model layer RDMs result in subject-specific $\quad 430$ correlation values per layer (Figure $3 \mathrm{E}$ ). To account for different levels of noise ${ }^{431}$ in brain ROIs, we estimated the noise ceiling in EVC and IT 47, 48, and 432 normalized the fMRI ROI and model correlations with the corresponding noise ${ }^{433}$ ceiling [49]. Then the correlation time series (for MEG/Model comparisons) or ${ }^{434}$ correlation values (for fMRI/Model comparisons) were averaged over participant ${ }_{435}$ and tested against zero for statistical significance. $\quad 436$ 


\section{Statistical tests}

We used nonparametric statistical test methods which make no assumptions on $\quad 438$

the distribution of the data [50,51]. For statistical inference on the correlation ${ }_{439}$

time series, we used permutation-based cluster-size inference with null 440

hypothesis of zero. For statistical assessments of peak latencies, we 441

bootstrapped the subject-specific correlation time series for 1000 times to $\quad{ }_{442}$

estimate an empirical distribution over peak latencies $[12,29,43$.

\section{Acknowledgments}

We would like to thank Western BrainsCAN for the generous support of this ${ }_{445}$ research. Computational modeling was conducted on Compute Canada 446 resources.

\section{References}

1. Epstein R, Kanwisher N. A cortical representation the local visual environment. Nature. 1998;392(6676):598-601. doi:10.1038/33402.

2. Epstein R, Harris A, Stanley D, Kanwisher N. The parahippocampal place area: Recognition, navigation, or encoding? Neuron. 1999;23(1):115-125. doi:10.1016/S0896-6273(00)80758-8.

3. Martin Cichy R, Khosla A, Pantazis D, Oliva A. Dynamics of scene representations in the human brain revealed by magnetoencephalography and deep neural networks. NeuroImage. 2017;153:346-358.

doi:10.1016/j.neuroimage.2016.03.063.

4. Lowe MX, Rajsic J, Ferber S, Walther DB. Discriminating scene categories from brain activity within 100 milliseconds. Cortex; a journal 
devoted to the study of the nervous system and behavior.

2018;106:275-287. doi:10.1016/j.cortex.2018.06.006.

5. Henriksson L, Mur M, Kriegeskorte N. Rapid Invariant Encoding of Scene Layout in Human OPA. Neuron. 2019;103(1):161 - 171.e3. doi:https://doi.org/10.1016/j.neuron.2019.04.014.

6. Kanwisher N, McDermott J, Chun MM. The fusiform face area: A module in human extrastriate cortex specialized for face perception. Journal of Neuroscience. 1997;17(11):4302-4311. doi:10.1523/JNEUROSCI.17-11-04302.1997.

7. Dobs K, Isik L, Pantazis D, Kanwisher N. How face perception unfolds over time. Nature Communications. 2019;10(1):1258.

doi:10.1038/s41467-019-09239-1.

8. Grill-Spector K, Kushnir T, Hendler T, Edelman S, Itzchak Y, Malach R. A sequence of object-processing stages revealed by fMRI in the human occipital lobe. Human brain mapping. 1998;6(4):316-328.

9. Grill-Spector K, Kourtzi Z, Kanwisher N. The lateral occipital complex and its role in object recognition. Vision Research. 2001;41(10):1409-1422. doi:https://doi.org/10.1016/S0042-6989(01)00073-6.

10. Cichy RM, Pantazis D, Oliva A. Resolving human object recognition in space and time. Nature Neuroscience. 2014;17(3):455-462. doi:10.1038/nn.3635.

11. Isik L, Meyers EM, Leibo JZ, Poggio T. The dynamics of invariant object recognition in the human visual system. Journal of Neurophysiology. 2014;111(1):91-102. doi:10.1152/jn.00394.2013. 
12. Mohsenzadeh Y, Qin S, Cichy RM, Pantazis D. Ultra-rapid serial visual presentation reveals dynamics of feedforward and feedback processes in the ventral visual pathway. eLife. 2018;7. doi:10.7554/eLife.36329.

13. Mohsenzadeh Y, Mullin C, Lahner B, Cichy RM, Oliva A. Reliability and Generalizability of Similarity-Based Fusion of MEG and fMRI Data in Human Ventral and Dorsal Visual Streams. 2019;doi:10.3390/vision3010008.

14. Goodale MA, Milner AD. Separate visual pathways for perception and action. Trends in neurosciences. 1992;15(1):20-25. doi:10.1016/0166-2236(92)90344-8.

15. Ungerleider LG, Haxby JV. 'What' and 'where' in the human brain. Current opinion in neurobiology. 1994;4(2):157-165. doi:10.1016/0959-4388(94)90066-3.

16. Li P, Zhu S, Chen M, Han C, Xu H, Hu J, et al. A motion direction preference map in monkey V4. Neuron. 2013;78(2):376-388. doi:10.1016/j.neuron.2013.02.024.

17. DiCarlo JJ, Maunsell JHR. Anterior inferotemporal neurons of monkeys engaged in object recognition can be highly sensitive to object retinal position. Journal of neurophysiology. 2003;89(6):3264-3278. doi:10.1152/jn.00358.2002.

18. Chakravarthi R, Carlson TA, Chaffin J, Turret J, VanRullen R. The temporal evolution of coarse location coding of objects: Evidence for feedback.; 2014 .

19. Serre T, Oliva A, Poggio T. A feedforward architecture accounts for rapid categorization. Proceedings of the National Academy of Sciences. 2007;104(15):6424-6429. doi:10.1073/pnas.0700622104. 
20. DiCarlo JJ, Zoccolan D, Rust NC. How does the brain solve visual object recognition?; 2012.

21. Yamins DLK, Hong H, Cadieu CF, Solomon EA, Seibert D, DiCarlo JJ. Performance-optimized hierarchical models predict neural responses in higher visual cortex. Proceedings of the National Academy of Sciences. 2014;111(23):8619-8624. doi:10.1073/pnas.1403112111.

22. Tang H, Kreiman G. In: Recognition of occluded objects. Singapore: Springer-Verlag; 2017.

23. Rajaei K, Mohsenzadeh Y, Ebrahimpour R, Khaligh-Razavi SM. Beyond core object recognition: Recurrent processes account for object recognition under occlusion. PLOS Computational Biology. 2019;15(5):e1007001. doi:10.1371/journal.pcbi.1007001.

24. Kar K, Kubilius J, Schmidt K, Issa EB, DiCarlo JJ. Evidence that recurrent circuits are critical to the ventral stream's execution of core object recognition behavior. Nature Neuroscience. 2019;22(6):974-983. doi:10.1038/s41593-019-0392-5.

25. Kietzmann TC, Spoerer CJ, Sörensen LKA, Cichy RM, Hauk O, Kriegeskorte N. Recurrence is required to capture the representational dynamics of the human visual system. Proceedings of the National Academy of Sciences of the United States of America. 2019;116(43):21854-21863. doi:10.1073/pnas.1905544116.

26. Yamins D, Hong H, Cadieu C, Dicarlo JJ. Hierarchical Modular Optimization of Convolutional Networks Achieves Representations Similar to Macaque IT and Human Ventral Stream. NIPS. 2013;

27. Khaligh-Razavi SM, Kriegeskorte N. Deep Supervised, but Not Unsupervised, Models May Explain IT Cortical Representation. PLoS Computational Biology. 2014;10(11). doi:10.1371/journal.pcbi.1003915. 
28. Cox DD, Dean T. Neural networks and neuroscience-inspired computer vision; 2014. Available from:

http://www.ncbi.nlm.nih.gov/pubmed/25247371.

29. Cichy RM, Khosla A, Pantazis D, Torralba A, Oliva A. Comparison of deep neural networks to spatio-temporal cortical dynamics of human visual object recognition reveals hierarchical correspondence. Scientific Reports. 2016;6(1):1-13. doi:10.1038/srep27755.

30. Cichy RM, Kaiser D. Deep Neural Networks as Scientific Models; 2019.

31. Güçlü U, van Gerven MAJ. Deep neural networks reveal a gradient in the complexity of neural representations across the ventral stream. Journal of Neuroscience. 2015;35(27):10005-10014.

doi:10.1523/JNEUROSCI.5023-14.2015.

32. Mohsenzadeh Y, Mullin C, Lahner B, Oliva A. Emergence of Visual Center-Periphery Spatial Organization in Deep Convolutional Neural Networks. Scientific Reports. 2020;10(1):1-8.

doi:10.1038/s41598-020-61409-0.

33. Cichy RM, Roig G, Andonian A, Dwivedi K, Lahner B, Lascelles A, et al.. The Algonauts Project: A Platform for Communication between the Sciences of Biological and Artificial Intelligence; 2019.

34. Makhzani A, Shlens J, Jaitly N, Goodfellow I, Frey B. Adversarial Autoencoders. 2015;

35. Goodfellow IJ, Pouget-Abadie J, Mirza M, Xu B, Warde-Farley D, Ozair S, et al. Generative Adversarial Nets;. Available from: http://www.github.com/goodfeli/adversarial.

36. Tzourio-Mazoyer N, Landeau B, Papathanassiou D, Crivello F, Etard O, Delcroix N, et al. Automated anatomical labeling of activations in SPM 
using a macroscopic anatomical parcellation of the MNI MRI

single-subject brain. NeuroImage. 2002;15(1):273-289.

doi:10.1006/nimg.2001.0978.

37. Kriegeskorte N. Representational similarity analysis - connecting the branches of systems neuroscience. Frontiers in Systems Neuroscience. 2008;2(NOV):4. doi:10.3389/neuro.06.004.2008.

38. Kriegeskorte N, Kievit RA. Representational geometry: Integrating cognition, computation, and the brain; 2013.

39. Cadena SA, Denfield GH, Walker EY, Gatys LA, Tolias AS, Bethge M, et al. Deep convolutional models improve predictions of macaque V1 responses to natural images. PLOS Computational Biology. 2019;15(4):1-27. doi:10.1371/journal.pcbi.1006897.

40. Tadel F, Baillet S, Mosher JC, Pantazis D, Leahy RM. Brainstorm: A User-Friendly Application for MEG/EEG Analysis. Computational Intelligence and Neuroscience. 2011;2011:879716. doi:10.1155/2011/879716.

41. Taulu S, Simola J. Spatiotemporal signal space separation method for rejecting nearby interference in MEG measurements. Physics in Medicine \& Biology. 2006;51(7):1759.

42. Carlson T, Tovar DA, Alink A, Kriegeskorte N. Representational dynamics of object vision: The first $1000 \mathrm{~ms}$. Journal of Vision. 2013;13(10):1-1. doi:10.1167/13.10.1.

43. Pantazis D, Fang M, Qin S, Mohsenzadeh Y, Li Q, Cichy RM. Decoding the orientation of contrast edges from MEG evoked and induced responses. NeuroImage. 2018;180:267-279. doi:10.1016/j.neuroimage.2017.07.022. 
44. Cao Q, Shen L, Xie W, Parkhi OM, Zisserman A. VGGFace2: A dataset for recognising faces across pose and age;. Available from: http://www.robots.ox.ac.uk/.

45. Russakovsky O, Deng J, Su H, Krause J, Satheesh S, Ma S, et al. ImageNet Large Scale Visual Recognition Challenge. International Journal of Computer Vision. 2015;115(3):211-252. doi:10.1007/s11263-015-0816-y.

46. Zhou B, Lapedriza A, Khosla A, Oliva A, Torralba A. IEEE Transactions on Pattern Analysis and Machine Intelligence Places: A 10 million Image Database for Scene Recognition. 1109;doi:10.1109/TPAMI.2017.2723009.

47. Nili H, Wingfield C, Walther A, Su L, Marslen-Wilson W, Kriegeskorte N. A Toolbox for Representational Similarity Analysis. PLOS Computational Biology. 2014;10(4):1-11. doi:10.1371/journal.pcbi.1003553.

48. Oliva A. In: Gist of the Scene. vol. 696; 2005. p. 251-256.

49. Khaligh-Razavi SM, Cichy RM, Pantazis D, Oliva A. Tracking the Spatiotemporal Neural Dynamics of Real-world Object Size and Animacy in the Human Brain. Journal of Cognitive Neuroscience. 2018;30(11):1559-1576. doi:10.1162/jocn_a_01290.

50. Maris E, Oostenveld R. Nonparametric statistical testing of EEG- and MEG-data. Journal of Neuroscience Methods. 2007;164(1):177-190. doi:https://doi.org/10.1016/j.jneumeth.2007.03.024.

51. Pantazis D, Nichols TE, Baillet S, Leahy RM. A comparison of random field theory and permutation methods for the statistical analysis of MEG data. NeuroImage. 2005;25(2):383-394.

doi:10.1016/j.neuroimage.2004.09.040. 